\title{
MicroRNAs in CSF as prodromal biomarkers for Huntington disease in the PREDICT-HD study
}

Eric R. Reed, MS, Jeanne C. Latourelle, DSc, Jeremy H. Bockholt, BS, Joli Bregu, MS, Justin Smock, MD, Jane S. Paulsen, PhD, and Richard H. Myers, PhD, PREDICT-HD CSF ancillary study investigators

Neurology ${ }^{\circledR}$ 2018;90:e264-e272. doi:10.1212/WNL.0000000000004844

\section{Abstract}

\section{Objective}

To investigate the feasibility of microRNA (miRNA) levels in CSF as biomarkers for prodromal Huntington disease (HD).

\section{Methods}

miRNA levels were measured in CSF from 60 PREDICT-HD study participants using the HTG protocol. Using a CAG-Age Product score, 30 prodromal HD participants were selected based on estimated probability of imminent clinical diagnosis of $\mathrm{HD}$ (i.e., low, medium, high; $\mathrm{n}=10$ / group). For comparison, participants already diagnosed $(n=15)$ and healthy controls $(n=15)$ were also selected.

\section{Results}

A total of 2,081 miRNAs were detected and 6 were significantly increased in the prodromal HD gene expansion carriers vs controls at false discovery rate $\mathrm{q}<0.05$ (miR-520f-3p, miR-135b-3p, miR-4317, miR-3928-5p, miR-8082, miR-140-5p). Evaluating the miRNA levels in each of the $\mathrm{HD}$ risk categories, all 6 revealed a pattern of increasing abundance from control to low risk, and from low risk to medium risk, which then leveled off from the medium to high risk and HD diagnosed groups.

\section{Conclusions}

This study reports miRNAs as CSF biomarkers of prodromal and diagnosed HD. Importantly, miRNAs were detected in the prodromal HD groups furthest from diagnosis where treatments are likely to be most consequential and meaningful. The identification of potential biomarkers in the disease prodrome may prove useful in evaluating treatments that may postpone disease onset.

\section{Clinicaltrials.gov identifier}

NCT00051324.

\author{
Correspondence \\ Dr. Myers \\ rmyers@bu.edu \\ or Dr. Paulsen \\ jane-paulsen@uiowa.edu \\ RELATED ARTICLE

\section{Editorial} \\ CSF microRNA in patients \\ with Huntington disease \\ Page 151
}




\section{Glossary}

$\mathrm{AD}=$ Alzheimer disease $\mathbf{C A G}=$ cytosine, adenine, guanine; $\mathbf{C A P}=$ CAG-Age Product $\mathbf{F D R}=$ false discovery rate; $\mathbf{H D}=$ Huntington disease; $\mathbf{L P}=$ lumbar puncture; miRNA = microRNA; PD = Parkinson disease.

Huntington disease (HD) is an inherited neurodegenerative disease typically diagnosed in midlife, ${ }^{1}$ although symptoms may appear as early as age 3 and as late as age 85 . The mutation responsible for $\mathrm{HD}$ is an expanded cytosine, adenine, guanine (CAG) trinucleotide repeat in the first exon of the huntingtin gene. ${ }^{2}$ Neuropathologic changes involving the accumulation of the huntingtin protein ${ }^{3}$ and the degeneration of neurons precede motor diagnosis, with up to half of striatal neurons lost before diagnosis. ${ }^{4}$ Volumetric changes in the striatum are evident as early as 2 decades prior to predicted diagnosis, ${ }^{5}$ indicating that neuropathologic changes occur many years prior to clinical motor manifestation and that effective therapeutics to prevent neurodegeneration would need to be administered long before clinical onset. The lack of validated biomarkers for onset and progression of neurodegeneration prior to clinical manifestation impedes the evaluation of preventive therapies.

MicroRNAs (miRNAs) are small noncoding ribose molecules with a bonded nucleotide base that negatively regulate mRNA levels in a sequence-specific manner, binding to the $3^{\prime}$-untranslated region to initiate cleavage or translational repression. ${ }^{6,7}$ miRNAs are abundant in the CNS, and assist in various neuronal processes such as synaptic development, maturation, and plasticity. ${ }^{8,9}$ Because they are encapsulated in small vesicles (either exosomes or microvesicles), ${ }^{10}$ and are associated with argonaute-2 (AGO2) proteins of the RNA-induced silencing complex, miRNAs resist degradation by ribonuclease. Mounting evidence suggests that disease-specific miRNA profiles can be detected in CSF in Parkinson disease (PD) and Alzheimer disease $(\mathrm{AD}){ }^{11-13}$

Studies of human HD prefrontal cortex identified 75 significantly altered miRNAs, ${ }^{14}$ including several that were associated with age at $\mathrm{HD}$ motor onset or the level of neuropathologic involvement in the striatum. ${ }^{15,16}$ Some miRNA levels were altered in brain samples of prodromal HD mHTT carriers. ${ }^{16}$ Although there is evidence of altered miRNA levels in plasma samples of prodromal HD, the changes were subtle and not sufficiently sensitive for an effective biomarker. ${ }^{17}$ We therefore sought to assess the presence of miRNAs in CSF from HD prodromal individuals as a biomarker of neurodegeneration prior to diagnosis.

\section{Methods}

\section{Study design and participants}

The PREDICT-HD study is a prospective observational study with 32 international sites conducted from
September 2002 to July 2014. All PREDICT-HD participants had genetic testing prior to study enrollment. A total of 1,078 CAG-expanded (CAG >35; 64\% female) individuals prior to motor diagnosis of $\mathrm{HD}$ were enrolled in this study. As healthy controls, 304 non-CAG-expanded siblings were also included ( $65 \%$ female). Annual assessments in the domains of motor, cognitive, psychiatric, functioning, and brain imaging were obtained with collection of DNA, blood, saliva, and urine. The goal of PREDICT-HD was to find predictive markers for motor manifestation (clinical diagnosis) of $\mathrm{HD}$.

\section{Standard protocol approvals, registrations, and patient consents}

All participants gave informed written consent prior to study participation, and all study procedures were approved by each site's respective institutional review board.

\section{CSF sample acquisition}

CSF acquisition was added to the PREDICT-HD protocol at the end of the study at 6 sites. All participants underwent screening for the lumbar puncture (LP) the day prior to sample acquisition so that biospecimens would be collected after fasting and that screening blood sample laboratories could be conducted. Exclusion criteria for LP were (1) use of anticoagulant medication (i.e., warfarin, heparin) or antiplatelets (aspirin) within 14 days; (2) unable to fast for 8 hours; (3) any acute or chronic infection; (4) history of any chronic inflammatory disorder; (5) unstable medical or psychiatric disorder, disease, or illness; and (6) abnormalities in blood-based screening (e.g., abnormalities in prothrombin time, partial thromboplastin time, or low platelets). In a sterile environment, a Sprotte 24-G atraumatic spinal needle was used after adequate local anesthesia was administered. The site coordinator recorded the time for each component of the protocol. The first 1-2 mL of CSF from the first syringe was immediately sent at room temperature for basic CSF analyses conducted locally within 4 hours (i.e., for cell count, erythrocytes, total protein, and glucose). Remaining CSF was transferred to 15 $\mathrm{mL}$ conical polypropylene tubes at room temperature, mixed gently by inverting 3-4 times, and then centrifuged at $2,000 \mathrm{~g}$ for 10 minutes. Aliquots of $1.5 \mathrm{~mL}$ of supernatant were transferred to precooled $2-\mathrm{mL}$ microcentrifuge tubes, and stored at $-80^{\circ} \mathrm{C}$ until shipment on dry ice to the $\mathrm{Na}$ tional Institute of Neurological Disorders and Stroke biorepository. Over $77 \%$ of the samples in this study were collected by an internist at the University of Iowa (J.S.) and the remaining samples were collected from 5 sites in the PREDICT-HD CSF ancillary study. 


\section{Samples}

CSF samples for 60 participants were chosen by the PREDICT-HD Data Management Team. ${ }^{18,19}$ All samples were blinded by a unique code specific for this substudy. The samples included 15 participants clinically diagnosed with $\mathrm{HD}$ according to traditional criteria with diagnostic confidence level of 4 on the Unified Huntington's Disease Rating Scale, ${ }^{20}$ 30 participants determined to be prodromal gene expansion carriers for HD, and 15 healthy controls. Disease burden in the prodromal participants was determined by calculation of the CAG-Age Product $(\mathrm{CAP}=$ age $\times[\mathrm{CAG}-33.66]),{ }^{21}$ developed to reflect age-adjusted cumulative exposure to the effects of mutant huntingtin.

\section{miRNA preprocessing and quantification}

Fifteen microliters of CSF was processed for miRNA levels using the HTG Molecular Diagnostics miRNA whole transcriptome protocol HTG EdgeSeq system (htgmolecular. com/products/htg-edg-system-edgeseq). This process includes specific probes for 2,083 miRNAs, producing both raw small-RNA sequencing files and prequantified data. A maximum of 24 samples can be processed in a single run and samples were randomly assigned to each of 3 batches. Raw sequencing files were processed and eventually used for differential analyses. Initial checks for sample quality, as well as adapter sequence identification, was performed using FastQC (version 0.11.3, bioinformatics.babraham.ac.uk/projects/ fastqc/). For each sample, low-quality reads were removed using FastX (version 0.0.14, hannonlab.cshl.edu/fastx_toolkit/) FASTQ Quality Filter, using a quality score of $80 \%$. TruSeq Adapter Index 2 adapter sequence 9 (5'-GATCGGAAGAGCACACGTCTGAACTCCAGTCACCGATGTA TCTCGTATGCCGTCTTCTGCTTG-3') was removed from each read using Cutadapt (version 1.7.1), removing reads with fewer than 15 remaining nucleotides. Reads with the same sequence were combined using FastX (version 0.0.14) Collapser, reporting the number of duplicated reads per sequence. ${ }^{22}$ Reads were aligned to human genome version hg19 using Bowtie (version 1.1.1), allowing for 0 mismatches. ${ }^{23}$ Bam files were converted to bed files using bedtools (version 2.25.0) bamToBed. ${ }^{24}$ miRNAs were defined as reads aligning within \pm 4 bases from the start coordinate of annotated miRNAs from mirBase (version 20), filtered for the 2,083 probes. ${ }^{25}$ miRNA reads were counted using GenomicRanges (version 1.22.4) $\mathrm{R}$ package, removing reads greater than 27 bases. ${ }^{26}$ Of the 2,083 probes, we were able to count at least one read across all samples for 2,082 miRNAs. One miRNA was removed due to low expression (mean raw counts $<2$ across all samples). Therefore, differential analysis includes 2,081 individual miRNAs.

\section{Statistical analysis}

All analysis was carried out using $\mathrm{R}$ (version 3.2.2). Counts were normalized using the DESeq2/variance stabilization transformation in DESeq2 (version 1.10.1). ${ }^{27}$ These values were then adjusted for batch effects from their sequencing run, using ComBat (version 3.18.0). ${ }^{28}$ Unless otherwise stated, expression values reported in this article are count values, after transformation on a $\log _{2}$ scale. Sample-level quality control was conducted across all samples. All differential expression analyses were carried out with linear models using miRNA expression as the outcome variable.

False discovery rate (FDR) q values were calculated from nominal $p$ values using the Benjamini-Hochberg procedure, performed by first ordering the $p$ values, where the smallest $p$ value has a rank of 1 . Each $p$ value is then transformed by taking the product of the $p$ value and the total number of tests, and then dividing by the $p$ value ranking. Finally, the FDR $q$ values are assigned as the cumulative minimum of this new set, ordered by the reverse ranking of the original $p$ value. ${ }^{29}$ Inadequate power precluded analyses comparing expression of individual miRNAs to CAG repeat size in the 45 participants with HD.

\section{Sample-level quality control}

Outlier samples were detected via qualitative assessment of plots of the first 2 principal components of expression values across all samples. After initial outlier samples were removed, the first 2 principal components of the remaining samples were replotted and the remaining samples were reevaluated for outliers. After 2 iterations of this process, no additional samples were removed.

\section{Diagnosed HD vs controls}

Differential expression analysis between diagnosed $\mathrm{HD}$ and controls was performed using both the complete set of miRNAs as well as a subset of 16 miRNAs previously reported ${ }^{16}$ as differentially expressed between postmortem $\mathrm{HD}$ and control participants. In each model, age was included as a covariate.

\section{Ordinal scales of prodromal HD progression}

In order to explore the relationship of miRNA expression with estimated risk of clinical HD diagnosis, we assigned ordinal values to each clinical group. The following values were assigned: 0 to control, 1 to low risk, 2 to medium risk, 3 to high risk, and 4 to diagnosed manifest HD participants. Age was not included as a covariate in these models because it is a factor in assigning HD prodromal staging.

\section{Hierarchical clustering of diagnosed HD and controls}

Hierarchical clustering was carried out on diagnosed HD and controls, using a subset of miRNAs determined to be significantly differentially expressed (FDR q value $<0.1$ ) between the 2 groups. Euclidean distance with the Ward agglomerative method was used to cluster both the samples and miRNAs.

\section{Results}

\section{Differential analysis of miRNA expression in CSF between diagnosed HD and controls}

In order to evaluate altered miRNA expression in HD CSF, we performed differential expression in 2,081 miRNA probes 
that passed expression filtering, quantified from small-RNA sequencing using the HTG EdgeSeq system. Of the 60 samples processed, 56 passed quality control filtering, including 14 controls, 10 low risk, 8 medium risk, 10 high risk, and 14 diagnosed HD (table 1).

The initial analysis compared diagnosed HD to controls. After normalization and batch correction, miRNAs were tested independently using multivariate linear modeling, adjusting for age. Of the 2,081 miRNAs, 25 reached FDR significance $\mathrm{q}$ value $<0.1$, and 6 reached FDR significance $q$ value $<0.05$. In all 25 of these miRNAs, expression was upregulated in $\mathrm{HD}$ and 14 miRNAs had greater than 2-fold changes in expression $\left(\log _{2} \mathrm{FC}>1\right)$ in $\mathrm{HD}$ compared to control participants (table 2 ). The extent to which these 25 miRNAs separated HD cases from controls was further explored via hierarchical clustering, which revealed a clear partition between cases and controls, with all but $3 \mathrm{HD}$ samples and 3 control samples clustering within their group (figure 1).

None of the 16 miRNAs previously identified ${ }^{16}$ to be differentially expressed between postmortem $\mathrm{HD}$ and control brains reached statistical significance when performing FDR corrections for either the full set 2,081 miRNA or the candidate set of 16 miRNAs, though 4 miRNAs reached nominal significance ( $p$ value $<0.05$, table e-1, links.lww.com/ WNL/A52).

\section{Analysis of miRNA expression and estimated risk of HD diagnosis}

In order to evaluate the association between miRNA expression and progression in prodromal to diagnosed $\mathrm{HD}$, we assigned each group an ordinal variable, 0 to 4 , where 0 was assigned to controls, 4 to diagnosed HD

Table 1 Sample information before and after sample-level quality control

\begin{tabular}{|c|c|c|c|c|c|}
\hline & \multirow[b]{2}{*}{ Control } & \multicolumn{3}{|c|}{ Prodromal HD } & \multirow[b]{2}{*}{ Diagnosed HD } \\
\hline & & Low risk & Medium risk & High risk & \\
\hline \multicolumn{6}{|c|}{ Before sample quality control } \\
\hline $\mathbf{n}$ & 15 & 10 & 10 & 10 & 15 \\
\hline Age, y, mean (SD) & $45.91(13.98)$ & $31.21(9.89)$ & $38.93(9.33)$ & $51.22(15.89)$ & $55.94(8.69)$ \\
\hline CAG, mean (SD) & $20.53(4.1)$ & $41.6(1.78)$ & $42.4(1.84)$ & $43(4.08)$ & $42(1.46)$ \\
\hline \multicolumn{6}{|l|}{ Sex, n (\%) } \\
\hline Male & $7(46.67)$ & $5(50.00)$ & $5(50.00)$ & $5(50.00)$ & $5(33.33)$ \\
\hline Female & $8(53.33)$ & $5(50.00)$ & $5(50.00)$ & $5(50.00)$ & $10(66.67)$ \\
\hline \multicolumn{6}{|l|}{ Batch, n (\%) } \\
\hline 1 & $6(40.00)$ & $4(40.00)$ & $4(40.00)$ & $5(50.00)$ & $5(33.33)$ \\
\hline 2 & $6(40.00)$ & $4(40.00)$ & $4(40.00)$ & $4(40.00)$ & $6(40.00)$ \\
\hline 3 & $3(20.00)$ & $2(20.00)$ & $2(20.00)$ & $1(10.00)$ & $4(26.67)$ \\
\hline \multicolumn{6}{|c|}{ After sample quality control } \\
\hline n & 14 & 10 & 8 & 10 & 14 \\
\hline Age, y, mean (SD) & $45.36(14.33)$ & $31.21(9.89)$ & $39.85(10.13)$ & $51.22(15.89)$ & $55.51(8.85)$ \\
\hline CAG, mean (SD) & $20.71(4.2)$ & $41.6(1.78)$ & $42.38(2.07)$ & $43(4.08)$ & $42.14(1.41)$ \\
\hline \multicolumn{6}{|l|}{ Sex } \\
\hline Male & $6(42.86)$ & $5(50.00)$ & $4(50.00)$ & $5(50.00)$ & $5(35.71)$ \\
\hline Female & $8(57.14)$ & $5(50.00)$ & $4(50.00)$ & $5(50.00)$ & 9 (64.29) \\
\hline \multicolumn{6}{|l|}{ Batch, n (\%) } \\
\hline 1 & $6(42.86)$ & $4(40.00)$ & $4(50.00)$ & $5(50.00)$ & $4(28.57)$ \\
\hline 2 & $5(35.71)$ & $4(40.00)$ & $4(50.00)$ & $4(40.00)$ & $6(42.86)$ \\
\hline 3 & $3(21.43)$ & $2(20.00)$ & $0(0.00)$ & $1(10.00)$ & $4(28.57)$ \\
\hline
\end{tabular}


Table 2 Differentially expressed microRNAs (miRNAs) between diagnosed Huntington disease (HD) and controls

\begin{tabular}{|c|c|c|c|c|}
\hline miRNA & $\begin{array}{l}\text { Mean } \\
\text { expression }\end{array}$ & $\log F C$ & $p$ Value & $\begin{array}{l}\text { FDR } \\
\text { q value }\end{array}$ \\
\hline miR-520f-3p & 4.47 & 1.24 & 0.00005 & 0.040 \\
\hline miR-135b-3p & 3.53 & 1.16 & 0.00007 & 0.040 \\
\hline miR-4317 & 6.03 & 1.20 & 0.00008 & 0.040 \\
\hline miR-3928-5p & 6.37 & 0.98 & 0.00008 & 0.040 \\
\hline miR-8082 & 3.30 & 1.42 & 0.00013 & 0.049 \\
\hline miR-140-5p & 6.19 & 0.65 & 0.00014 & 0.049 \\
\hline miR-509-3-5p & 5.04 & 1.36 & 0.00020 & 0.055 \\
\hline miR-6516-5p & 4.06 & 1.50 & 0.00021 & 0.055 \\
\hline miR-455-3p & 3.76 & 0.95 & 0.00030 & 0.059 \\
\hline miR-6838-3p & 4.31 & 1.05 & 0.00030 & 0.059 \\
\hline miR-552-5p & 3.64 & 1.21 & 0.00033 & 0.059 \\
\hline miR-761 & 3.47 & 0.95 & 0.00037 & 0.059 \\
\hline miR-4659a-5p & 4.87 & 1.18 & 0.00037 & 0.059 \\
\hline miR-4781-5p & 6.15 & 0.92 & 0.00041 & 0.061 \\
\hline miR-4462 & 4.73 & 1.05 & 0.00053 & 0.074 \\
\hline miR-132-5p & 5.34 & 0.90 & 0.00058 & 0.074 \\
\hline miR-6818-5p & 3.81 & 1.03 & 0.00060 & 0.074 \\
\hline miR-34c-3p & 3.05 & 0.86 & 0.00072 & 0.083 \\
\hline miR-4724-3p & 6.87 & 1.08 & 0.00076 & 0.083 \\
\hline miR-4307 & 5.97 & 0.95 & 0.00089 & 0.090 \\
\hline miR-6874-5p & 3.98 & 1.10 & 0.00091 & 0.090 \\
\hline miR-5581-3p & 3.76 & 0.95 & 0.00101 & 0.094 \\
\hline miR-6807-5p & 5.09 & 0.90 & 0.00104 & 0.094 \\
\hline miR-922 & 3.13 & 1.28 & 0.00112 & 0.094 \\
\hline miR-1322 & 3.73 & 1.33 & 0.00113 & 0.094 \\
\hline
\end{tabular}

Abbreviation: $F D R=$ false discovery rate.

Results of differential expression of miRNAs between 14 diagnosed HD and 14 control participants. Shown are the 6 miRNAs with FDR q values $<0.05$ and an additional 19 with $q<0.1$, ordered by nominal $p$ value. These $p$ values reflect the coefficient for HD status, adjusted for participant age in a multivariate linear model. FDR q values are calculated using the Benjamini-Hochberg procedure for the set of 2,081 miRNAs tested. The mean expression values are calculated from the DESeq2/variance stabilized and batch-corrected values across all 28 participants. The values for logFC specify the $\log _{2}$ transformation of the fold change of miRNA expression of participants with HD vs controls.

participants, and 1-3 to each of the prodromal groups. Linear modeling of the 2,081 expressed miRNAs across the 56 samples revealed no miRNAs that reached FDR significance, although 16 had nominal $p$ values $<0.005$ (table 3 ). These 16 miRNAs included the top 5 significantly differentially expressed $(\mathrm{q}<0.05)$ in the HD vs control analysis: miR-520f-3p, miR-135b-3p, miR-4317, miR-3928-5p, miR-8082. Boxplots of the distribution of expression across each group for all 6 miRNAs differentially expressed between HD vs control participants are shown in figure 2. For each of these miRNAs, the direction of the $\log _{2} \mathrm{FC}$ between adjacent nominal groups is consistent with the direction of altered expression seen between HD vs controls.

None of the candidate miRNAs reported previously ${ }^{16}$ as differentially expressed in HD vs control prefrontal cortex reached FDR $\mathrm{q}<0.1$, and only 2 reached nominal significance (miR-132-3p, $p<0.017$, miR-5695, $p<0.05$, table e-2, links. lww.com/WNL/A53).

\section{Discussion}

This analysis reports the assessment of miRNAs in HD CSF as a biomarker for HD. We evaluated the differential levels of miRNAs for individuals diagnosed with HD vs controls as well as the relationship of miRNA levels among gene expansion-positive prodromal individuals with varying estimated risk of diagnosis (table 1). We first sought to distinguish miRNAs that characterize diagnosed HD using a discovery set of 2,081 miRNAs. Six miRNAs were differentially found in diagnosed HD vs control CSF (FDR q $<0.05$ ) and an additional 19 at FDR q $<0.1$ (table 2). All of the miRNAs were upregulated in HD CSF. However, none of the miRNAs that we had previously identified with differential levels in diagnosed HD vs control prefrontal cortex brain samples ${ }^{16}$ were found to be different in these early diagnosed HD CSF samples.

When examining the association of miRNA expression to an ordinal scale of diagnosis risk, or time to diagnosis, where 0 was assigned to controls, 4 to diagnosed HD participants, and 1-3 to each prodromal group with decreasing proximity to (or risk of) diagnosis, 16 miRNAs had nominal $p<0.005$ (FDR $<0.326$ ), including the top 5 differentially expressed in diagnosed HD vs controls FDR q $<0.05$ (table 3). When we plotted the 6 FDR significant miRNAs, we observed a consistent pattern of association between miRNA expression across prodromal groups. Specifically, miRNA increases from control to low risk and increases again from low risk to medium risk but then appears to remain elevated across the medium risk to high risk and HD diagnosed groups (figure 2).

While this study shows altered miRNA expression in HD CSF, similar studies have been performed for $\mathrm{AD}$ and PD. A study $^{11}$ using small-RNA sequencing to quantify 2,228 miRNAs in $69 \mathrm{AD}, 67 \mathrm{PD}$, and 78 control participants reported differential expression of 41 miRNAs in $A D$ vs controls and 17 miRNAs in PD vs controls. A similar study $^{13}$ using TaqMan low-density array human miRNA panels to quantify 746 exosomal miRNAs in CSF across 28 


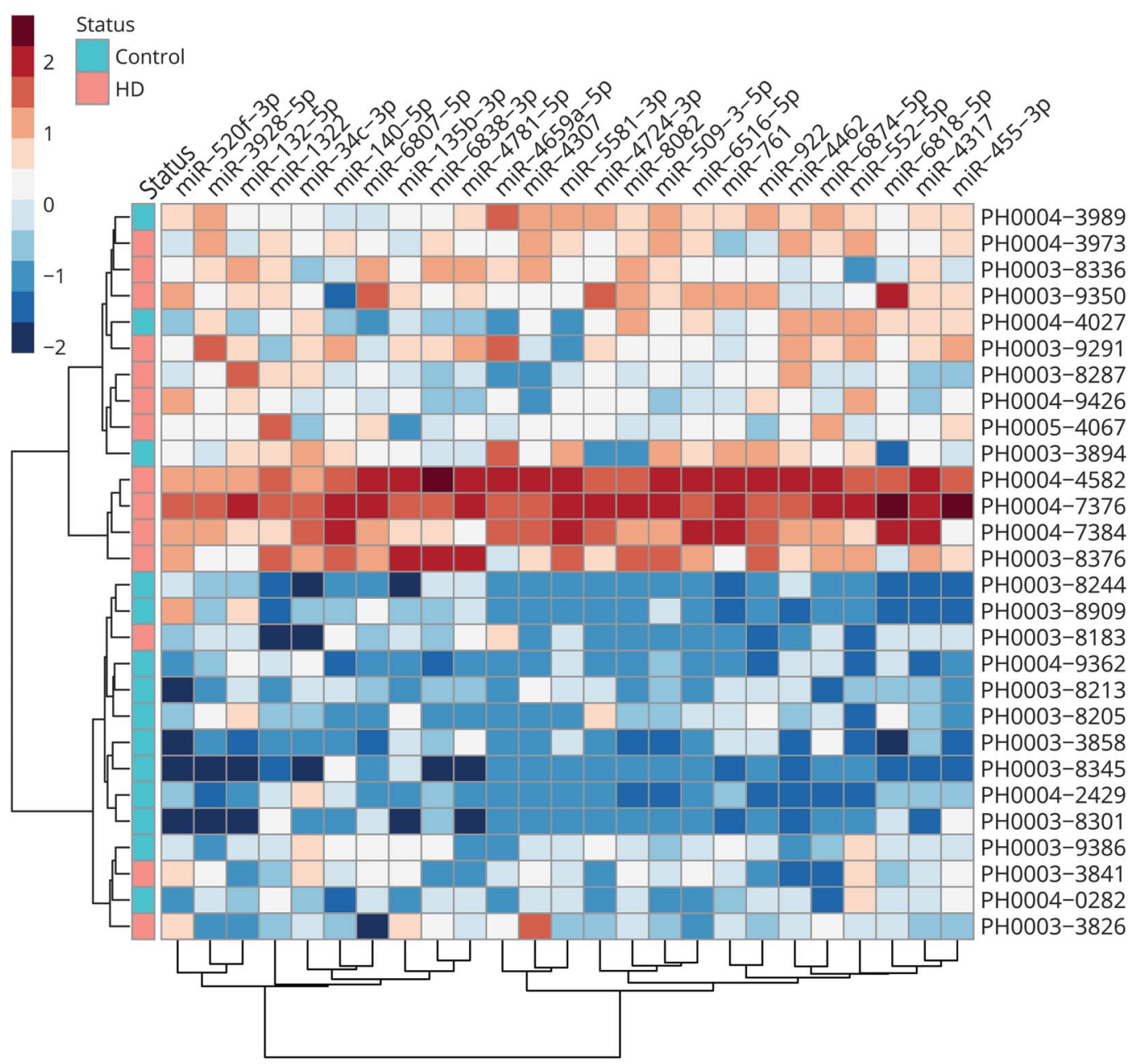

Hierarchal clustering of 14 diagnosed Huntington disease cases and 14 controls presented on the X axis defined by the color at the top of the figure, using the top 25 most differentially expressed miRNAs presented on the $Y$ axis (table 2). Samples and miRNAs have been clustered based on their normalized expression. Colors in this heatmap reflect miRNA-wise $z$ score transformation of normalized expression where darker shades of red represent increased levels and darker shades of blue represent decreased levels.

$\mathrm{AD}, 47 \mathrm{PD}$, and 27 control participants reported differential expression of 27 miRNAs in $\mathrm{AD}$ vs controls and 6 miRNAs in PD vs controls. These studies show concordance with our results, most notably miR-132-5p, identified in the PD analysis, ${ }^{11}$ as well as in both the $\mathrm{AD}$ and $\mathrm{PD}$ analyses. ${ }^{13}$ In our analysis, miR-132-5p was differentially expressed in diagnosed HD vs controls (table 2), as well as nominally associated with ordinal categorization of prodromal HD progression $(p=0.035$, FDR $=0.33)$. miR-132$3 p$ was included in the set of miRNAs that were differentially regulated in HD brain. ${ }^{16}$ Of these 16 miRNAs, miR$132-3 p$ had the second lowest nominal $p$ value when comparing diagnosed HD vs control CSF $(p=0.025$, FDR $=0.15$; table $\mathrm{e}-1$, links.lww.com/WNL/A52), as well as the lowest nominal $p$ value for the ordinal relationship ( $p=$ 0.020 , FDR $=0.27)$. Also identified in the $\mathrm{AD}$ analysis, ${ }^{11}$ miR-760 was one of the top 16 miRNAs in our ordinal analysis $(p=0.0038, \mathrm{FDR}=0.36$; table 3$)$.
Several points can be made from these studies. First, we did not see a strong relationship between miRNA levels that distinguish HD from control in brain with the miRNA levels that distinguish HD from control in CSF. The process by which miRNAs are released into CSF is still not wellunderstood, and it may be that miRNAs released into CSF are derived from the degeneration of neurons as the integrity of the neuronal cell membrane is lost while the predominant differential miRNA levels seen in $\mathrm{HD}$ brain may instead reflect miRNAs found in non-neuronal cell types (microglia, astrocytes, and oligodendrocytes).

Second, the pattern for miRNA increase present for the earliest prodromal stages of HD may be important for future clinical trials as those miRNAs may reflect changes occurring in the brain that echo effects of the initial neurodegeneration seen in $\mathrm{HD}$, long before clinical diagnosis. A panel of miRNAs may provide insight into whether 
Table 3 MicroRNA (miRNA) expression association with ordinal categories of control, prodromal, and diagnosed Huntington disease (HD)

\begin{tabular}{|c|c|c|c|c|}
\hline & Mean expression & $\log F C$ & $p$ Value & FDR q value \\
\hline miR-18b-5p & 4.95 & 0.23 & 0.00052 & 0.326 \\
\hline $\operatorname{miR}-135 b-3 p^{a}$ & 4.34 & 0.20 & 0.00086 & 0.326 \\
\hline miR-875-3p & 6.28 & 0.21 & 0.00091 & 0.326 \\
\hline $\operatorname{miR}-3928-5 p^{a}$ & 6.40 & 0.16 & 0.00095 & 0.326 \\
\hline miR-520f-3p & 4.14 & 0.18 & 0.00146 & 0.326 \\
\hline $\operatorname{miR}-4317^{a}$ & 6.30 & 0.20 & 0.00229 & 0.326 \\
\hline miR-4252 & 5.48 & 0.14 & 0.00317 & 0.326 \\
\hline miR-4499 & 4.75 & 0.22 & 0.00336 & 0.326 \\
\hline miR-6838-3p & 4.51 & 0.20 & 0.00337 & 0.326 \\
\hline $\operatorname{miR}-8082^{a}$ & 4.86 & 0.22 & 0.00341 & 0.326 \\
\hline $\operatorname{miR}-760$ & 4.48 & 0.12 & 0.00379 & 0.326 \\
\hline miR-4723-3p & 4.25 & -0.09 & 0.00409 & 0.326 \\
\hline miR-4491 & 5.41 & 0.22 & 0.00433 & 0.326 \\
\hline miR-4327 & 6.33 & 0.17 & 0.00452 & 0.326 \\
\hline miR-335-3p & 5.20 & 0.14 & 0.00488 & 0.326 \\
\hline miR-7705 & 5.69 & 0.23 & 0.00497 & 0.326 \\
\hline
\end{tabular}

Abbreviation: FDR = false discovery rate.

Results of univariate linear modeling of miRNA expression vs ordinal categories of risk of diagnosis. Shown are the 16 miRNAs with the lowest nominal $p$ values. These $p$ values reflect the coefficient for ordinal group membership. FDR q values are calculated using the Benjamini-Hochberg procedure for the set of 2,081 miRNAs tested. The mean expression values are calculated from the DESeq2/variance stabilized and batch-corrected values across all 56 participants. The logFC values represent the estimated change in miRNA expression between 2 adjacent ordinal groups, calculated as the log 2 transformation of the fold change between 2 adjacent study groups on the ordinal scale.

a Significantly differentially expressed between HD and controls (table 2).

treatments are preventing the initiation of the degenerative process in HD clinical trials that seek to prevent early damaging effects of the HTT gene on the integrity of the brain. These findings show particular promise since very few baseline/cross-sectional measures have detected differences between the low-risk/far from diagnosis prodromal group and controls. To our knowledge, only emotion recognition ${ }^{30}$ and striatal volumes ${ }^{31}$ from MRI are reportedly different between controls and prodromal participants who are furthest from HD diagnosis. Biomarkers to detect and track the earliest measures of disease will become important in future clinical trials of preventive therapies.

Finally, we recognize that the sample size of 60 studied here may not have sufficient power to detect all of the miRNAs that are altered in diagnosed or prodromal HD relative to controls. Additional studies of larger cohorts throughout the continuum of the disease spectrum, and studies of samples taken longitudinally, will almost certainly reveal additional important insights into the utility of miRNA measures in CSF as biomarkers for prodromal HD. We also recognize the imprecision of the prodromal staging variable CAP. Although research has validated the utility of this variable, ${ }^{19}$ biomarker studies are likely to improve as the prodromal stages of HD are further characterized and subjected to clinimetrics. Generalizability of our findings with regards to sex, ethnicity, race, and potential environmental factors is unknown since the PREDICT study recruited all participants with regards to HD risk. Confidence in the findings might be strengthened since the control group represented siblings whose genetic test revealed a normal CAG length, offering control for some variation in individual differences. An additional limitation of our study is that the prodromal groups are partially defined by age, with those further from diagnosis being younger than those nearer to diagnosis risk. Consequently, adjusting for age across these groups is problematic. Effects of age on the levels of miRNAs may be a source of bias that we are not able to consider in a study of this size. Finally, we recognize that 3 of the HD cases did not cluster with the others and cluster as controls in figure 1 . These may be a consequence of assay failure or other unknown factors that alter the levels of miRNAs in CSF. Additional work to 
Figure 2 Plots of microRNAs (miRNAs) across categories of control, prodromal, and diagnosed Huntington disease (HD)

A. miR-520f-3p

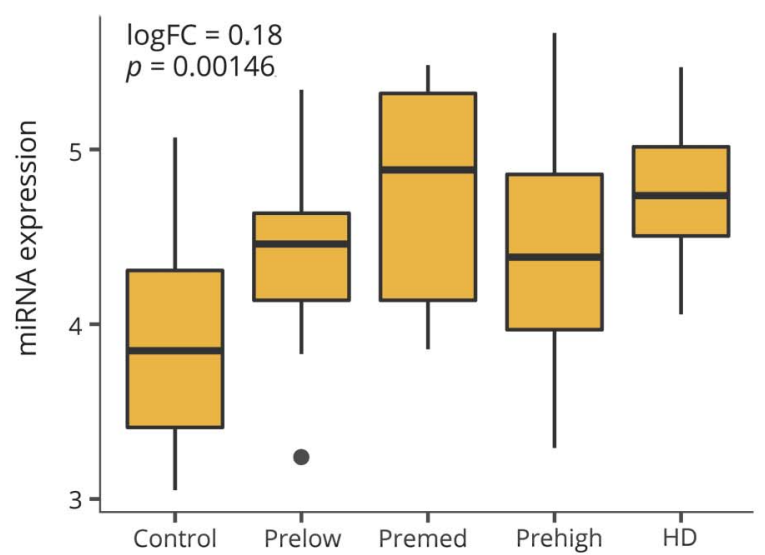

C. $\operatorname{miR}-4317$

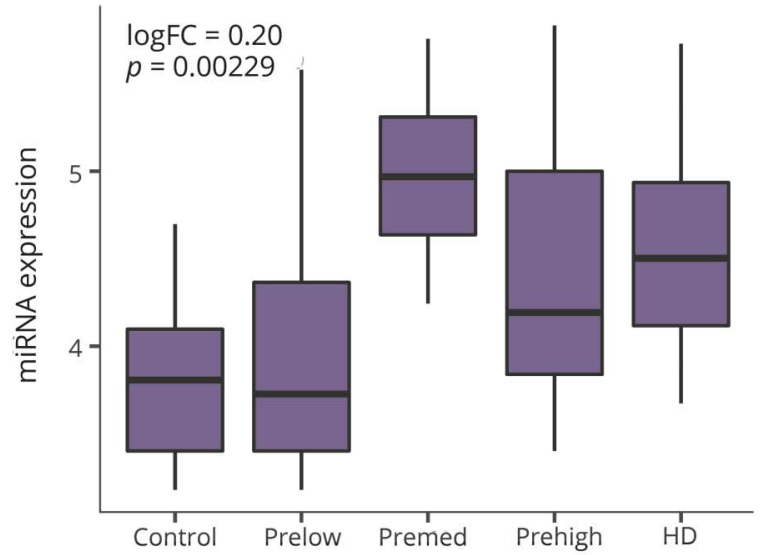

E. miR-8082

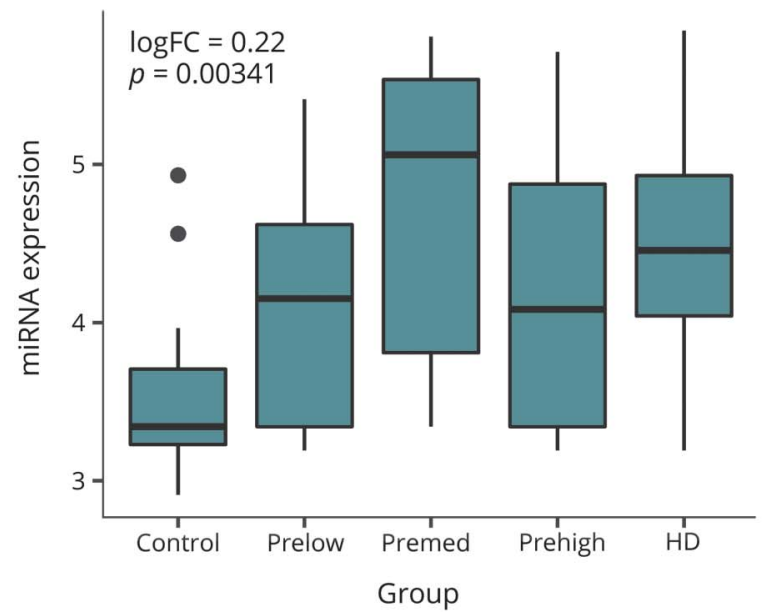

B. $\operatorname{miR}-135 b-3 p$

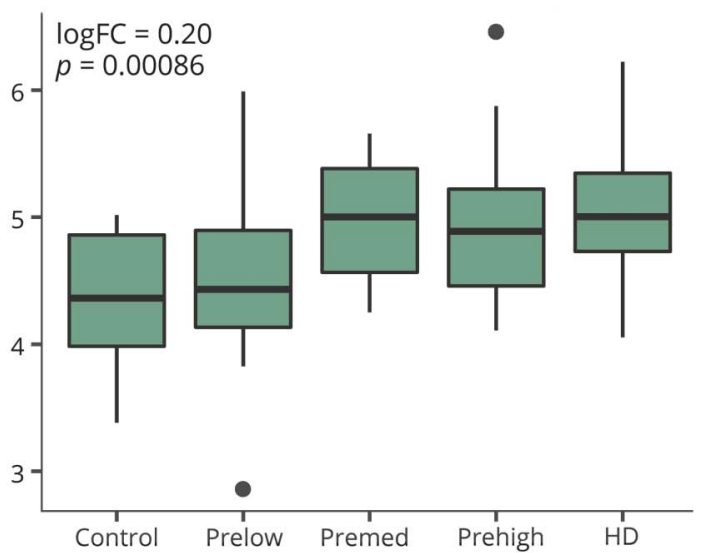

D. miR-3928-5p

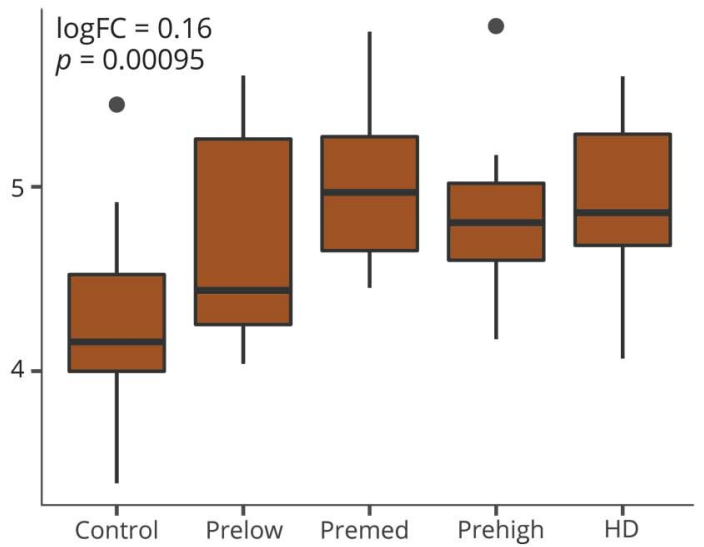

F. miR-140-5p

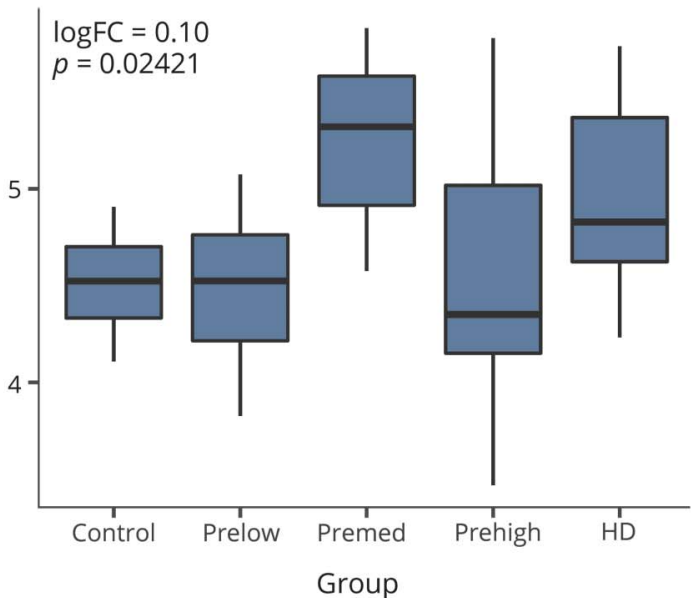

Boxplots of the distribution of DESeq2/variance stabilized and batch-corrected expression among the 5 ordinal groups (risk of diagnosis of HD) for each of the 6 miRNAs differentially expressed between HD and control participants (table 2; A, 50f-3p; B, 135b-3p; C, 4317; D, 3928-5p; E, 8082; F, 140-5p). $p$ Values and logFC values are the same as in table 3. The low-risk, medium-risk, high-risk, and diagnosed HD groups are synonymous with the far from onset, middle from onset, near onset, and symptomatic HD groups. 
determine the sources of miRNA variation is likely to improve the efficacy of these measures.

\section{Author contributions}

R.H.M. and J.S.P. conceived and designed the study. E.R.R., J. C.L., J.H.B., J.B., J.S., J.S.P., and R.H.M. acquired, analyzed, or interpreted the data. E.R.R., R.H.M., and J.S.P. drafted the manuscript. E.R.R., J.C.L., J.H.B., J.B., J.S., J.S.P., and R.H.M. provided critical revision of the manuscript for important intellectual content. E.R.R., J.C.L., and J.H.B. conducted data and statistical analysis. R.H.M. and J.S.P. obtained funding and provided study supervision.

\section{Acknowledgment}

The authors thank the PREDICT-HD sites, the study participants, the National Research Roster for Huntington Disease Patients and Families, the Huntington's Disease Society of America, the Huntington Study Group, and the European Huntington's Disease Network.

\section{Study funding}

Supported by the Jerry McDonald HD Research Fund and by the NIH, National Institute of Neurologic Disorders and Stroke grant (3R01-NS073947) awarded to Richard H. Myers; and by the NIH, National Institute of Neurologic Disorders and Stroke grants (5R01NS040068, 5R01NS054893, 5U01NS082089) and the CHDI Foundation, Inc. (A6266; A2015) awarded to Jane S. Paulsen. This publication was supported by the National Center for Advancing Translational Sciences and the NIH. The content is solely the responsibility of the authors and does not necessarily represent the official views of the NIH.

\section{Disclosure}

The authors report no disclosures relevant to the manuscript. Go to Neurology.org/N for full disclosures.

Received November 8, 2016. Accepted in final form September 29, 2017.

\section{References}

1. Myers RH. Huntington's disease genetics. NeuroRx 2004;1:255-262.

2. MacDonald ME, Ambrose CM, Duyao MP, et al. A novel gene containing a trinucleotide repeat that is expanded and unstable on Huntington's disease chromosomes. Cell 2016;72:971-983.

3. Gómez-Tortosa E, Macdonald ME, Friend JC, et al. Quantitative neuropathological changes in presymptomatic Huntington's disease. Ann Neurol 2001;49:29-34.
4. Vonsattel JP, Myers RH, Stevens TJ, Ferrante RJ, Bird ED, Richardson EP Jr. Neuropathological classification of Huntington's disease. J Neuropathol Exp Neurol 1985; 44:559-577.

5. Aylward EH, Sparks BF, Field KM, et al. Onset and rate of striatal atrophy in preclinical Huntington disease. Neurology 2004;63:66-72.

6. Bartel DP. MicroRNAs: genomics, biogenesis, mechanism, and function. Cell 2004; 116:281-297.

7. Bartel DP. MicroRNAs: target recognition and regulatory functions. Cell 2009;136: 215-233.

8. Schratt GM, Tuebing F, Nigh EA, et al. A brain-specific microRNA regulates dendritic spine development. Nature 2006;439:283-289.

9. Cao X, Yeo G, Muotri AR, Kuwabara T, Gage FH. Noncoding RNAs in the mammalian central nervous system. Annu Rev Neurosci 2006;29:77-103.

10. Arroyo JD, Chevillet JR, Kroh EM, et al. Argonaute2 complexes carry a population of circulating microRNAs independent of vesicles in human plasma. Proc Natl Acad Sci USA 2011;108:5003-5008.

11. Burgos K, Malenica I, Metpally R, et al. Profiles of extracellular miRNA in cerebrospinal fluid and serum from patients with Alzheimer's and Parkinson's diseases correlate with disease status and features of pathology. PLoS One 2014;9:e94839.

12. Kumar S, Reddy PH. Are circulating microRNAs peripheral biomarkers for Alzheimer's disease? Biochim Biophys Acta 2016;1862:1617-1627.

13. Gui Y, Liu H, Zhang L, Lv W, Hu X. Altered microRNA profiles in cerebrospinal fluid exosome in Parkinson disease and Alzheimer disease. Oncotarget 2015;6:37043-37053.

14. Hoss AG, Kartha VK, Dong X, et al. MicroRNAs located in the hox gene clusters are implicated in Huntington's disease pathogenesis. PLoS Genet 2014;10:e1004188.

15. Hadzi TC, Hendricks AE, Latourelle JC, et al. Assessment of cortical and striatal involvement in 523 Huntington disease brains. Neurology 2012;79:1708-1715.

16. Hoss AG, Labadorf A, Latourelle JC, et al. miR-10b-5p expression in Huntington's disease brain relates to age of onset and the extent of striatal involvement. BMC Med Genomics 2015;8:10.

17. Hoss AG, Lagomarsino VN, Frank S, Hadzi TC, Myers RH, Latourelle JC. Study of plasma-derived miRNAs mimic differences in Huntington's disease brain. Mov Disord 2015;30:1961-1964.

18. Paulsen JS, Hayden M, Stout JC, et al. Preparing for preventive clinical trials: the PREDICT-HD study. Arch Neurol 2006;63:883-890.

19. Paulsen JS, Long JD, Ross CA, et al. Prediction of manifest Huntington disease with clinical and imaging measures: a 12 -year prospective observational study. Lancet Neurol 2014;13:1193-1201.

20. Huntington Study Group. Unified Huntington's Disease Rating Scale: reliability and consistency.Mov Disord 1996;11:136-142.

21. Zhang Y, Long JD, Mills JA, Warner JH, Lu W, Paulsen JS; PREDICT-HD Investigators and Coordinators of the Huntington Study Group. Indexing disease progression at study entry with individuals at risk for Huntington disease. Am J Med Genet B Neuropsychiatr Genet 2011;156:751-763.

22. Martin M. Cutadapt removes adapter sequences from high-throughput sequencing reads. EMBnet.journal 2011;17:10.

23. Langmead B, Trapnell C, Pop M, Salzberg SL. Ultrafast and memory-efficient alignment of short DNA sequences to the human genome. Genome Biol 2009;10:R25.

24. Quinlan AR, Hall IM. BEDTools: a flexible suite of utilities for comparing genomic features. Bioinformatics 2010;26:841-842

25. Kozomara A, Griffiths-Jones S. MiRBase: annotating high confidence microRNAs using deep sequencing data. Nucleic Acids Res 2014;42:68-73.

26. Lawrence $M$, Huber $W$, Pagès $H$, et al. Software for computing and annotating genomic ranges. PLoS Comput Biol 2013;9:e1003118.

27. Love MI, Huber W, Anders S. Moderated estimation of fold change and dispersion for RNA-seq data with DESeq2. Genome Biol 2014;15:550.

28. Johnson WE, Li C, Rabinovic A. Adjusting batch effects in microarray expression data using empirical Bayes methods. Biostatistics 2007;8:118-127.

29. Benjamini Y, Hochberg Y. Controlling the false discovery rate: a practical and powerful approach to multiple testing. J R Stat Soc 1995;57:289-300.

30. Stout JC, Paulsen JS, Queller S, et al. Neurocognitive signs in prodromal Huntington disease. Neuropsychology 2011;25:1-14.

31. Paulsen JS, Nopoulos PC, Aylward E, et al. Striatal and white matter predictors of estimated diagnosis for Huntington disease. Brain Res Bull 2010;82:201-207. 


\section{MicroRNAs in CSF as prodromal biomarkers for Huntington disease in the PREDICT-HD study}

Eric R. Reed, MS, Jeanne C. Latourelle, DSc, Jeremy H. Bockholt, BS, Joli Bregu, MS, Justin Smock, MD, Jane S. Paulsen, PhD, and Richard H. Myers, PhD, The PREDICT-HD CSF ancillary study investigators

Cite as: Neurology ${ }^{\circledR}$ 2018;90:e264-e272. doi:10.1212/WNL.0000000000004844

\author{
Correspondence \\ Dr. Myers \\ rmyers@bu.edu \\ or Dr. Paulsen \\ jane-paulsen@uiowa.edu
}

\section{Study question}

Can microRNA (miRNA) levels in CSF serve as biomarkers of neurodegeneration in Huntington disease (HD) prodromal individuals?

\section{Summary answer}

miRNAs are effective CSF biomarkers for prodromal HD long before diagnosis.

\section{What is known and what this article adds}

Volumetric changes (in the striatum) are often the earliest indicators of $\mathrm{HD}$ onset and progression. However, there is a distinct lack of validated biomarkers. This study reveals CSF biomarkers that could help detect and prevent HD.

\section{Participants and setting}

CSF miRNA levels from 60 PREDICT-HD participants were measured; 30 participants with prodromal HD were selected. The rest were part of a comparison study, where 15 individuals were healthy and 15 had HD.

\section{Design, size, and duration}

This was a prospective observational study comprising 32 international sites from September 2002 to July 2014. In total, 1,078 CAG-expanded individuals were enrolled prior to HD diagnosis as well as 305 non-CAG-expanded siblings as healthy controls. CSF miRNA levels were measured via the HTG molecular diagnostics miRNA whole transcriptome protocol, which includes specific probes for 2,083 miRNAs. Patients with prodromal HD were selected based on estimation of imminent clinical HD diagnosis based on a CAG-Age Product (CAP) score.

\section{Primary outcomes, risks, and exposures}

Differential miRNA expression levels were determined for both individuals with diagnosed HD and controls, and the relationship of miRNA levels among prodromal individuals with different risk diagnoses.

\section{Main results and the role of chance}

Of the 2,081 detected miRNAs, differential expression of 6 miRNAs (miR-520f-3p, miR-135b-3p, miR-4317, miR-3928-5p, miR-8082, and miR-140-5p) was significantly higher in the prodromal HD gene expansion carriers than in the controls $(q<0.05)$. This increase
Plots of microRNAs (miRNAs) across categories of control prodromal, and diagnosed Huntington disease (HD)

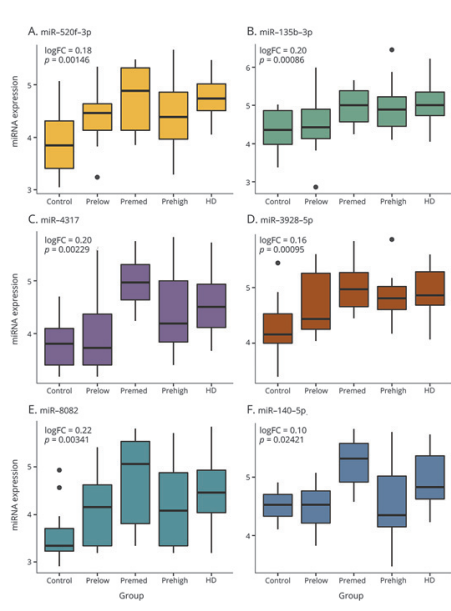

in expression was significant in the low-HD risk group compared to the control and in the medium-HD risk group compared to the low$\mathrm{HD}$ risk group. However, there were no such observations between the medium to high-HD risk and $\mathrm{HD}$-diagnosed groups.

Bias, confounding, and other reasons for caution The sample size may not have had sufficient detection power to identify all differentially expressed miRNAs in diagnosed or prodromal HD compared to controls. CAP scores may not be accurate as a prodromal HD readout. Age adjustment across groups was problematic as prodromal groups were partially defined by age, as those further from diagnosis were younger than those closer to diagnosis risk. Furthermore, effects of age on miRNA levels may serve as a source of bias. Three HD cases did not cluster, possibly owing to assay failure.

\section{Generalizability to other populations}

Given that the PREDICT study recruited all participants with regard to $\mathrm{HD}$ risk, generalizability of the findings with regard to sex, ethnicity, race, and potential environmental factors may be limited.

\section{Study funding/potential competing interests}

The study was funded by a group of foundation and government grants. Go to Neurology.org/N for full disclosures.

A draft of the short-form article was written by E. Feric, a writer with Editage, a division of Cactus Communications. The authors of the full-length article and the journal editors edited and approved the final version. 


\section{Neurology}

\section{MicroRNAs in CSF as prodromal biomarkers for Huntington disease in the PREDICT-HD study}

Eric R. Reed, Jeanne C. Latourelle, Jeremy H. Bockholt, et al.

Neurology 2018;90;e264-e272 Published Online before print December 27, 2017

DOI 10.1212/WNL.0000000000004844

\section{This information is current as of December 27, 2017}

\section{Updated Information \& Services}

References

Citations

Subspecialty Collections

Permissions \& Licensing

Reprints including high resolution figures, can be found at: http://n.neurology.org/content/90/4/e264.full

This article cites 31 articles, 4 of which you can access for free at: http://n.neurology.org/content/90/4/e264.full\#ref-list-1

This article has been cited by 2 HighWire-hosted articles: http://n.neurology.org/content/90/4/e264.full\#\#otherarticles

This article, along with others on similar topics, appears in the following collection(s):

Case control studies

http://n.neurology.org/cgi/collection/case_control_studies

Cerebrospinal Fluid

http://n.neurology.org/cgi/collection/cerebrospinal_fluid

Huntington's disease

http://n.neurology.org/cgi/collection/huntingtons_disease

Information about reproducing this article in parts (figures,tables) or in its entirety can be found online at:

http://www.neurology.org/about/about_the_journal\#permissions

Information about ordering reprints can be found online:

http://n.neurology.org/subscribers/advertise

Neurology ${ }^{\circledR}$ is the official journal of the American Academy of Neurology. Published continuously since 1951, it is now a weekly with 48 issues per year. Copyright Copyright ( 2017 The Author(s). Published by Wolters Kluwer Health, Inc. on behalf of the American Academy of Neurology.. All rights reserved. Print ISSN: 0028-3878. Online ISSN: 1526-632X.

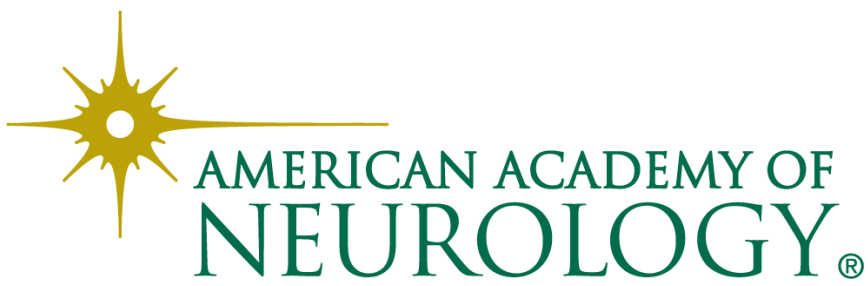

